Bull. Mater. Sci., Vol. 13, No. 3, June 1990, pp. 211-216. (C) Printed in India.

\title{
Positron annihilation studies on nasicon analogues containing cation vacancies
}

\author{
V SREERAMALU, H R SREEPAD, A CHANDRASHEKARA, \\ V RAVINDRACHARY and S GOPAL
}

Department of Studies in Physics, University of Mysore, Manasagangotri, Mysore 570006, India

MS received 11 December 1989

\begin{abstract}
Positron annihilation studies were carried out on the Nasicon analogue $\mathrm{Na}_{2}(\mathrm{La}, \mathrm{Al}) \mathrm{Zr}\left(\mathrm{PO}_{4}\right)_{3}$ compound for three different concentrations $(2.2,2.8$ and 5.2 by wt. $\%$ of $\mathrm{ZrO}_{2}$ in the nutrient. Angular correlation study of annihilated photons reveals that the defect concentration is maximum for $2.8(\mathrm{wt} . \%)$ of $\mathrm{ZrO}_{2}$. Further, positron lifetime studies indicate that the positrons are trapped at cation vacancies. Application of a twostate trapping model to this system made it possible to evaluate the lifetime of positrons in the Bloch state and of positrons trapped at cation vacancies.
\end{abstract}

Keywords. Nasicon analogue; positron annihilation spectroscopy; cation vacancies; trapping rate.

\section{Introduction}

Since high sodium ion mobility was discovered in the $\mathrm{Na}_{1+x} \mathrm{Zr}_{2} \mathrm{Si}_{x} \mathrm{P}_{3-x} \mathrm{O}_{12}(0 \leqslant x \leqslant 3)$ (Hong 1976) system, the Nasicon (sodium superionic conductor) compounds have attracted wide scientific interest as potential solid electrolytes in sodium-sulphur battery systems and in other electrochemical devices. The ionic conductivity of these materials are found to be almost equivalent to that of $\mathrm{Na}-\beta$-alumina (Goodenough et al 1976). Since then, material scientists are searching for new Nasicon materials with simpler structures, stoichiometric in composition and easily synthesized as single crystals. Most of these materials are isostructural with $\mathrm{Na}_{3} \mathrm{Sc}_{2} \mathrm{P}_{3} \mathrm{O}_{12}$ crystals and hence these are popularly known as Nasicon analogues. In these new Nasicon analogues, the ionic conductivity and optical properties depend mainly on the type of rare earth cation and the crystalline structure (Byrappa 1986). In the basic structure of Nasicon, the $\mathrm{Na}^{+}$ions are constrained to move in the three-dimensional network of the oxide frame. The crystalline framework is constituted by $\mathrm{PO}_{4}$ tetrahedra sharing corners with $\mathrm{ZrO}_{6}$ octahedra. This basic arrangement is preserved for a wide range of compositions, but the ionic conductivity strongly depends on the composition.

The most common defect structures in ionic crystals are vacancy pairs (Schottky defects) and vacancy-interstitial pairs (Frenkel defects). The concentration of these defects can be altered at low temperatures by suitable chemical substitution (Worell 1977). The crucial phenomenon responsible for superionic properties is probably the formation of vacancies in the bulk and interstitials at the surface. The role played by such vacancies in the bulk and their conduction mechanism can be understood by studying their concentration and other related physical properties by using positron annihilation spectroscopy (PAS).

PAS has proved to be a powerful technique in studying the nature of defects present in condensed matter as compared to other conventional methods. Positron 
angular correlation experiments on ionic crystals generally suggest that the momentum distribution depends mainly on the negative ion. The sensitivity of positrons is related to the trapping of positrons at defects. Vacancy defects create an effective negative charge and if the electrical potential created by the defect is strong enough, it will give rise to a bound state of the positron because of change in the relative electron density at or close to a defect (Dupasquier 1979). Annihilation characteristics are changed in such a way that the response can be used in principle to determine whether the positron is annihilated in the perfect part of the crystal or at the defect. The earlier angular correlation studies (Vorobev et al 1972; Ramaswamy and Nagarajan 1976) on ionic crystals clearly show the sensitivity of positrons to ionic distortions and their dependence on the FWHM (full width at half maximum $-\Gamma_{1 / 2}$ ). Also, in $\mathrm{KCl}-\mathrm{KBr}$ mixed crystals they observed that the $\Gamma_{1 / 2}$ becomes minimum for maximum concentration of defects at a given composition. In recent years there have been a number of measurements of positron annihilation for various nonmetallic crystals. Complicated lifetime spectra have been observed for ionic solids and the effects of lattice defects on lifetimes have been deduced. In these ionic solids the shortest lifetime component $\tau_{1}$ ranges from $140-350 \mathrm{ps}$ and is normally associated with annihilation in the bulk of the crystal. A second lifetime component $\tau_{2}$ was observed to range from $300-780$ ps (Bisi et al 1963; Bertolaccini et al 1971). The cause for several lifetimes and decay modes is not established unequivocally (Dupasquier 1979). In analogy with metals, the second lifetime component is associated with the trapping of positrons by lattice defects. The most likely candidate in ionic crystals would appear to be a cation vacancy. Because of redistribution of the electrons near the vacancy, it is not clear whether one or two traps (one much weaker than the other) might be expected in our Nasicon analogue. Therefore, our experiments are prompted by the fact that cation vacancies are responsible for the ionic conduction in Nasicon analogues and various authors agree about the effect of vacancies on ionic conductivity and other related properties of Nasicon analogues.

\section{Experimental method}

Three Nasicon analogue $\mathrm{Na}_{2}(\mathrm{La}, \mathrm{Al}) \mathrm{Zr}\left(\mathrm{PO}_{4}\right)_{3}$ crystals of varying concentration $(2.2,2.8$ and $5.2 \mathrm{wt} . \%)$ of $\mathrm{ZrO}_{2}$ were received from the Minerological Institute, University of Mysore, Mysore. These were synthesized using hydrothermal techniques by varying the concentration of $\mathrm{ZrO}_{2}$ (by wt. $\%$ ) in the nutrient as described by Byrappa et al (1988). Later these crystals were powdered and pressed into pellets (dia: $10 \mathrm{~mm}$, thickness: $2-3 \mathrm{~mm}$, compactness: $85-90 \%$ ). The pressed pellets were sintered in a vacuum chamber $\left(10^{-2}\right.$ Torr) at $300^{\circ} \mathrm{C}$ for $30 \mathrm{~min}$ and quenched in air before carrying out the experiments.

Angular correlation of annihilated photons were measured at the Indira Gandhi Centre for Atomic Research, Kalpakkam. The apparatus used has a resolution of $0.6 \mathrm{mrad}$; a source ${ }^{22} \mathrm{Na}$ having a strength of $5 \mathrm{mCi}$ was used. The experimental details are given elsewhere (Sarkar et al 1982). Angular correlation spectra were obtained for three samples having $\mathrm{ZrO}_{2}$ concentrations of $2 \cdot 2,2.8$ and $5.2 \%$ by weight. Positron lifetime measurements (Gopinathan et al 1980) were performed with a fast-fast coincidence system using KL-236 plastic scintillators cum RCA8575 photomultiplier assembly. The time resolution of the spectrometer was $340 \mathrm{ps}$ 
(FWHM) for $40 \%{ }^{22} \mathrm{Na}$ energy gating. The instrumental resolution and the source correction were obtained from the known lifetime spectrum of a metal, fitted to the programme RESOLUTION. After source and background corrections, the lifetime components and their intensities of the system under study were resolved using the programme PATFIT (Kirkegaard et al 1988).

\section{Results and discussion}

The results of various experiments on ionic crystals reveal that the relative changes in the annihilation parameters observed are extremely strong in comparison with the molar fractions of defects present in the samples. This clearly indicates that the positrons are probably attracted and captured by some defect species. Hence the annihilation characteristics respond not to the average properties of the crystal but to the situation locally encountered by the positrons in the defect region (Dupasquier 1979). Brandt et al (1972) have shown that positrons are attracted by the free positive vacancies as well as vacancies associated with divalent impurities. The present positron angular correlation measurements on these samples of the Nasicon analogue as in figure 1 , show that the $\Gamma_{1 / 2}$ (FWHM) is minimum for the sample containing 2.8 (wt.\%) of $\mathrm{ZrO}_{2}$ and it is maximum for the other two compositions. It indicates that defect concentration is maximum for 2.8 (wt.\%) of $\mathrm{ZrO}_{2}$. The probable defects at this concentration are the interstitial-type as well as being cation vacancies. Further increase in the concentration shows that the defect concentration is minimum indicating that cation vacancies increase when compared to interstitial-type defects.

The results of lifetime studies and their corresponding intensities are shown in table 1, with errors for three different concentrations of $\mathrm{ZrO}_{2}$ (wt.\%). The positron spectrum contains a tail having a mean life of a few nanoseconds and its intensity is equal to or greater than $2 \%$. This tail has been reported in table 1 and its origin is to be attributed to positron annihilation in regions other than the homogeneous interior of the crystal (Paulin and Ambrosino 1968). Positrons are essentially

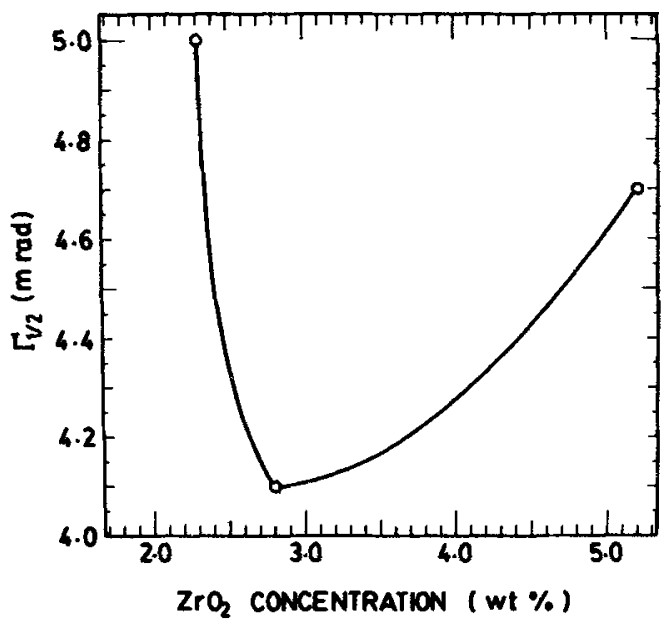

Figure 1. Variation of $\Gamma_{1 / 2}$ (FWHM) with $\mathrm{ZrO}_{2}$ concentration (wt.\%). 
Table 1. Positron lifetime results of $\mathrm{Na}_{2}(\mathrm{La}, \mathrm{Al}) \mathrm{Zr}\left(\mathrm{PO}_{4}\right)_{3}$.

\begin{tabular}{lcccccc}
\hline $\begin{array}{l}\text { Sample with } \mathrm{ZrO}_{2} \\
\text { (wt. \%) }\end{array}$ & $\begin{array}{c}\tau_{1} \\
(\mathrm{ps})\end{array}$ & $\begin{array}{c}\tau_{2} \\
(\mathrm{ps})\end{array}$ & $\begin{array}{c}\tau_{3} \\
(\mathrm{ps})\end{array}$ & $\begin{array}{c}I_{1} \\
(\%)\end{array}$ & $\begin{array}{c}I_{2} \\
(\%)\end{array}$ & $\begin{array}{c}I_{3} \\
(\%)\end{array}$ \\
\hline $2 \cdot 2$ & $247 \pm 14$ & $424 \pm 47$ & $1529 \pm 205$ & $64 \pm 1$ & $33 \pm 1$ & $2 \pm 1$ \\
$2 \cdot 8$ & $273 \pm 4$ & $590 \pm 30$ & $7306 \pm 273$ & $82 \pm 2$ & $17 \pm 2$ & $1 \pm 0$ \\
$5 \cdot 2$ & $247 \pm 17$ & $442 \pm 36$ & $1622 \pm 206$ & $51 \pm 1$ & $46 \pm 1$ & $3 \pm 1$ \\
\hline
\end{tabular}

insensitive to regions of higher-than-average electron density as found at interstitial or small interstitial clusters. Thus the results of positron lifetime experiments on Nasicon analogues containing cation-vacancies that can trap positrons are analysed in terms of two-state trapping model as described by Siegel (1980). The two-state trapping model assumes that positrons exist in one of only two states in the material. The bulk or Bloch state and the defect-trapped state are in relative concentrations of $n_{b}(t)$ and $n_{t}(t)$ respectively with $n_{t}(0)=0$. These are described by the set of coupled differential equations:

$$
\begin{aligned}
& \frac{\mathrm{d} n_{b}}{\mathrm{~d} t}=-n_{b} \lambda_{b}-n_{b} k_{t}, \\
& \frac{\mathrm{d} n_{t}}{\mathrm{~d} t}=n_{b} k_{t}-n_{t} \lambda_{t},
\end{aligned}
$$

where $\lambda_{b}$ and $\lambda_{t}$ are the positron annihilation rates in the bulk and defect-trapped states respectively and $k_{t}$ is the total transition or trapping rate of the positron from the bulk to the defect-trapped state. The positron lifetimes in the two states are defined by

$$
\tau_{b}=\lambda_{b}^{-1} \text { and } \tau_{t}=\lambda_{t}^{-1} \text {. }
$$

The solutions of (1) and (2) readily yield the fraction of positrons present in the system at time $t$,

$$
\begin{aligned}
N(t) & =n_{b}(t)+n_{t}(t) \\
& =\left(1-I_{2}\right) \exp \left(-\lambda_{1} t\right)+I_{2} \exp \left(-\lambda_{1} t\right),
\end{aligned}
$$

where $\lambda_{1}, \lambda_{2}$ and $I_{2}$ are the decay rates and intensity that can be obtained from the deconvolution analysis of an observed positron lifetime spectrum. These experimentally observed parameters $\lambda_{1}, \lambda_{2}$ and $I_{2}$ are related to the physical quantities $\lambda_{b}, \lambda_{t}$ and $k_{t}$ by

$$
\left.\begin{array}{l}
\lambda_{b}=\left(1-I_{2}\right) \lambda_{1}+I_{2} \lambda_{2} \\
\lambda_{b}=\lambda_{1}-k_{t} \\
\lambda_{t}=\lambda_{2} \\
k_{t}=I_{2}\left(\lambda_{1}-\lambda_{2}\right) \\
k_{t}=\left(\lambda_{b}-\lambda_{t}\right) \frac{I_{2}}{1-I_{2}}
\end{array}\right]
$$

Using (4) and the related experimental values from table 1, the trapping rate $k_{t}$ is evaluated. The variation of trapping rate with concentration, as shown in figure 2 , clearly demonstrates that at $2.8 \mathrm{wt} . \%$ of $\mathrm{ZrO}_{2}$ the trapping rate is minimum indicating that the clusters of interstitial-type defects are at the maximum. Thus it is 
clear that for large concentrations, the cation vacancies are the maximum. Figure 3 shows the plot of $\lambda_{1}$ versus $k_{t}$ and it is seen that the experimental points lie on the straight line showing that the two-state trapping model assumption is valid. The intersection of the straight line with the $\mathrm{Y}$-axis yields the annihilation rate in the bulk, i.e. $\lambda_{b}$. The variation of $k_{t}$ with $I_{2} /\left(1-I_{2}\right)$ is shown in figure 4 . The slope of this straight line graph gives the value of $\left(\lambda_{b}-\lambda_{t}\right)$. Hence from this the rate of positrons trapped at the cation vacancies is determined. The above results suggest that the application of a two-state trapping model gives the evidence that positrons are trapped generally at cation vacancies in the superionic material.

\section{Conclusions}

(1) The lifetime of positrons in the bulk or Bloch state in Nasicon analogue is $278 \mathrm{ps}$.

(2) The lifetime of positrons trapped at cation vacancies is 348 ps.

(3) Positron annihilation has proved to be an invaluable method for studying certain aspects of metal physics. Nasicon compounds and their analogues have not

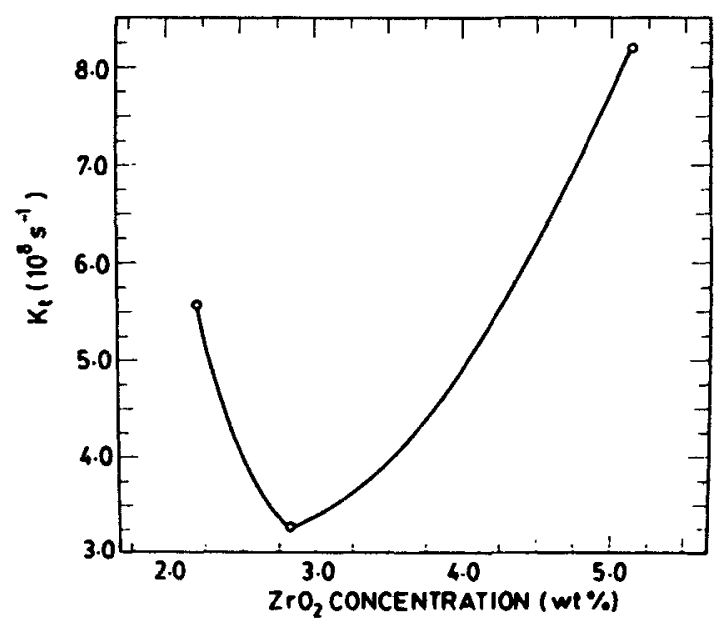

Figure 2. Variation of $K_{t}$ (trapping rate) with concentration of $\mathrm{ZrO}_{2}$ (wt. $\%$ ).

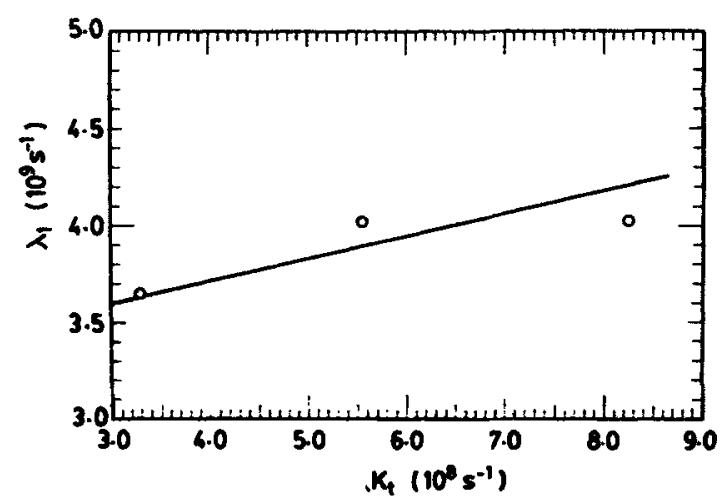

Figure 3. Variation of $\lambda_{1}$ (decay rate) with $K_{t}$. 


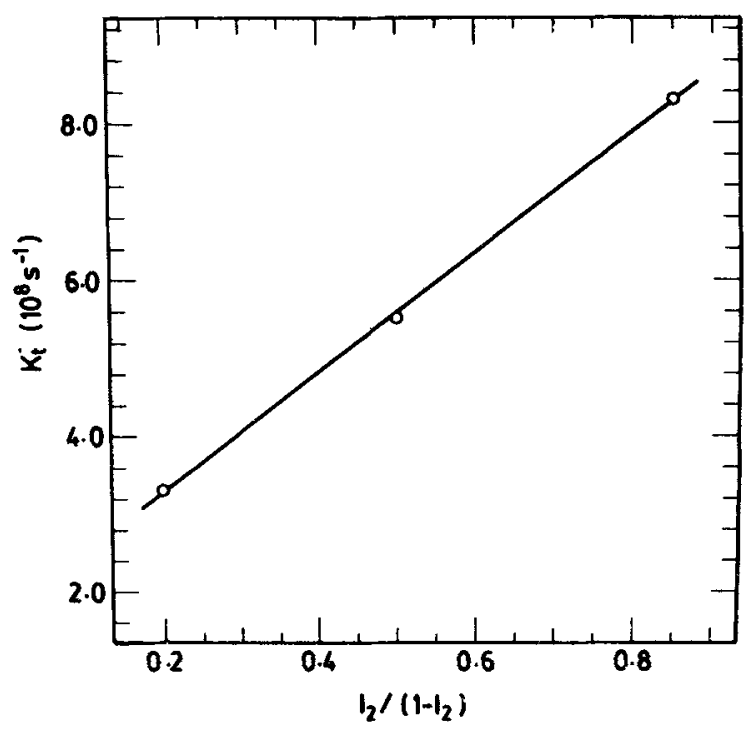

Figure 4. Variation of $K_{\mathrm{t}}$ with $I_{2} /\left(1-I_{2}\right)$.

yet been adequately explored using positrons. Our simple approach seems to satisfy some major characteristics of experimental results from positron annihilation study. Further positron studies in this area are unquestionably needed.

\section{Acknowledgements}

The authors are grateful to Dr B Viswanathan of the Indira Gandhi Centre for Atomic Research, Kalpakkam, for having provided facilities for carrying out the positron angular correlation experiments. The authors also gratefully acknowledge Dr K Byrappa of the Minerological Institute, University of Mysore, Mysore, for having provided the samples. VS and VR thank the University Grants Commission, New Delhi, for financial assistance.

\section{References}

Bertolaccini M, Bisi A, Gambarini G and Zappa L 1971 J. Phys. C4 734

Bisi A, Florentini A and Zappa L 1963 Phys. Rev. 1311023

Brandt W, Dupasquier A and Durr G 1972 Phys. Rev. B6 3156

Byrappa K 1986 Prog. Cryst. Growth Charact. 13163

Byrappa K, Gopalakrishna G S, Kulkarni A B and Desai N B 1988 J. Less Common Met. 1391

Dupasquier A 1979 Positron in solids (ed.) P Hautojarvi (Amsterdam: North Holland) p. 197

Goodenough J, Hong H Y P and Kaflas J A 1976 Mater. Res. Bull. 11203

Gopinathan K P, Sundar C S, Viswanathan B and Bharathi A 1980 Bull. Mater. Sci. 2207

Hong H Y P 1976 Mater. Res. Bull. 11173

Kirkegaard P, Pedersen N J and Eldrup M 1988 PATFIT Program of Riso National Laboratory Report, Denmark, M-2740 (1989)

Paulin R and Ambrosino G 1968 J. Phys. 29263

Ramaswamy P and Nagarajan T 1976 Physica B81 305

Sarkar S K, Sundar C S, Murthy N S, Bharathi A, Viswanathan B and Gopinathan K P 1982 J. Madras Uni. B45 380

Siegel R W 1980 Annu. Rev. Mater. Sci. 10393

Vorobev A A, Aretev K P and Vorobev S A 1972 Phys. Status Solidi B53 K133

Worell W L 1977 Solid electrolytes (ed.) S Geller (Heidelberg: Springer Verlag) p. 143 